\title{
Decreased Ferroportin in Hepatocytes Promotes Macrophages Polarize Towards an M2-Like Phenotype and Liver Fibrosis
}

\section{Chengyuan Cai}

Guangzhou Medical University

\section{Danning Zeng}

Guangzhou Medical University

\section{Qing Gao}

Infinitus (China) Company Ltd.

Lei Ma

Guangzhou Medical University

Bohang Zeng

Guangzhou Medical University

Yi Zhou ( $\nabla$ zhouyi0264@gzhmu.edu.cn )

Guangzhou Medical University

He Wang

Guangzhou Medical University

\section{Research Article}

Keywords: FPN1, Fibrosis, Macrophage, TGF- $\beta$, Liver.

Posted Date: February 3rd, 2021

DOl: https://doi.org/10.21203/rs.3.rs-150742/v1

License: (c) (i) This work is licensed under a Creative Commons Attribution 4.0 International License. Read Full License 


\section{Abstract}

Iron release from macrophages is closely regulated by the interaction of hepcidin, a peptide hormone produced by hepatocytes, with the macrophage iron exporter ferroportin (FPN1). However, the functions of FPN1 in hepatocyte secretion and macrophage polarization remain unknown. CD68 immunohistochemical staining and double immunofluorescence staining for $F 4 / 80$ and $K i 67$ in transgenic mouse livers showed that the number of macrophages in $\mathrm{FPN1}^{-/+}$and $\mathrm{FPN}^{-/-}$mouse livers was significantly increased compared to that in WT $\left(F P N^{+/+}\right)$mice. FPN1 downregulation in hepatic cells increased the levels of the M2 markers CD206, TGF- $\beta, V E G F, M M P-9$, Laminin, Collagen, IL-4 and IL-10. Furthermore, the expression of $C D 16 / 32$ and $i N O S$, as M1 markers, exhibited the opposite trend. Meanwhile, $a-S M A$ immunohistochemistry and Sirius red staining showed that the trend of liver fibrosis in $F P N 1^{-/-}$mice was more significant than that in control mice. Similarly, in vitro FPN1 knockdown in L02Sh/L02-SCR liver cell lines yielded similar results. Taken together, we demonstrated that downregulated FPN1 expression in hepatocytes can promote the proliferation and polarization of macrophages, leading to hepatic fibrosis. Above all, the FPN1 axis might provide a potential target for hepatic fibrosis.

\section{Introduction}

Hepatic fibrosis is an abnormal change in normal liver tissue caused by persistent necrosis, inflammation and the repair of fibrous connective tissue after chronic liver injury. As a chronic liver disease, it is characterized by hepatocyte injury and the excessive deposition of extracellular matrix. Meanwhile, liver fibrosis, as an early stage of liver cirrhosis, can also destroy normal tissue structure and cause serious damage to tissue function ${ }^{1}$.

Ferroportin1 (FPN1, SLC4OA1) is the only known membrane protein that transports iron out of cells ${ }^{2}$. It is highly expressed in cells involved in iron uptake, storage and reuse, such as duodenal epithelial cells, hepatocytes, reticuloendothelial macrophages and placental syncytiotrophoblasts ${ }^{3}$. In addition to being related to iron transport, FPN1 is closely related to inflammation. Studies have shown that inflammation can directly activate Toll-like receptors (TLRs) or induce the production of hepcidin through inflammatory factors such as interleukin-6(IL-6). Hepcidin can reduce the expression of FPN1 through the hepcidinFPN1 axis. Mice die in the embryonic stage when FPN1 is completely knocked out, but mice with specific knockout in hepatocytes can survive.

It has been reported that when one allele of the FPN1gene is mutated, an autosomal dominant genetic disorder called ferroportin disease (FD) occurs ${ }^{4,5}$. This mutation reduces iron transport, especially in reticuloendothelial macrophages, and eventually leads to iron accumulation in macrophages in the spleen, liver and bone. Due to the deficiency in FPN1 activity in hepatocytes, iron deposition in discrete hepatocytes in liver tissue is evident even in the early stage ${ }^{6}$. The clinical symptoms vary, but the overall manifestation is milder than that of classical hemochromatosis $(\mathrm{HC})$. Both classical forms of HFE-HC and FD are associated with antioxidant defense and organ fibrosis. However, the mechanism by which 
FD induces liver fibrosis remains unknown. In HC, but not in FD, a ubiquitination mutant of FPN1 caused by an FPN1 mutation results in a decrease in the sensitivity of the FPN1 protein on the cell surface to hepcidin and a reduction in the iron content in macrophages.

Macrophages are important components of nonspecific immunity. Macrophages (known as Kupffer cells in the liver) play a critical role in liver fibrosis. Liver contain tissue resident macrophages that are indispensable for tissue homeostasis. It has reported that the majority of tissue-resident macrophages are initially derived from the embryonic yolk-sac and maintain via self-renewal; ; however, this varies amongst tissues. Circulating monocytes contribute to the resident macrophage pool in some tissues, monocyte-derived macrophages (MdMs) redominantly enter tissues in states of tissue injury or inflammation ${ }^{8}$. Although tissue resident macrophages share functions like clearance of cellular debris and tissue remodeling, they can also exert specific tissue function ${ }^{9}$.

By regulating the activation or apoptosis of hepatic stellate cells and the formation and degradation of fibrocollagen, macrophages can promote or reverse the two-way regulation of liver fibrosis ${ }^{10,11}$. Based on their surface markers and the cytokines they secreted, macrophages can be divided into proinflammatory M1 and anti-inflammatory M2 phenotypes. M1 macrophages can induce high expression of nitric oxide synthase (iNOS), increase the expression of $C D 16 / 32$ on the membrane surface and promote the secretion of inflammatory cytokines, such as $I L-1 \beta, I L-6, I L-12 a, I L-12 \beta, T N F-a$ and IFN- $\gamma$. M2 macrophages secrete anti-inflammatory cytokines such as $I L-4, I L-10, T G F-a, T G F-\beta, V E G F$ and $C D 206^{12}$. In the acute stage of inflammation, M2 macrophages can reduce the secretion of proinflammatory cytokines and alleviate inflammation damage, but in the chronic stage of inflammation, M2 macrophages can secrete profibrotic factors, including typical $T G F-\beta$, which can promote the activation of myofibroblasts and the synthesis of extracellular matrix and promote the development of fibrosis.

The purpose of this study was to investigate the relationship between the expression of FPN1 in hepatocytes and hepatic fibrosis and the role of these hepatocytes in macrophage proliferation and polarization and to explore which factors are involved in these processes.

\section{Results}

FPN1 regulated macrophage proliferation and polarization. FPN1 knockout mice were used in this study (Fig. 1A-C). It has reported that the predicted molecular mass of purified human FPN1 is approximately 69-kDa. Yet, in mouse tissues, different FPN1 molecular weight (MW) forms have been detected ${ }^{13}$.As FPN1 expression was downregulated in liver cells, the iron content in liver cells was increased (Fig. 1D). To test whether silencing FPN1 in hepatic cells contributed to the changes in macrophages, double immunofluorescence staining for $F 4 / 80$ and $C D 68$ immunohistochemical staining and double immunofluorescence staining for $C D 68$ and Ki67 were performed in liver sections from WT $\left(F P N^{+/+}\right)$and FPN1 knockout mice (Fig. 1E and F). The results showed that the number of macrophages in $F P N 1^{-/+}$ and $F P N 1^{-/-}$mouse livers was significantly increased compared to that in $F P N^{+/+}$mouse livers (Fig. 1G-I). 
Macrophage polarization by was detected by immunofluorescence staining and flow cytometry in liver tissues from $\mathrm{FPN}^{+/+}, \mathrm{FPN1}^{-/+}$and $\mathrm{FPN1^{-/- }}$ mice. It was shown that macrophages in $\mathrm{FPN1^{-/- }}$ and $F P N 1^{-1+}$ mice were obviously polarized to the M2 subtype (Fig. 2A-C). Among all livers, the percentage $\mathrm{CD} 206$ in $\mathrm{FPN1}^{-/-}$mice was 3.94-fold and 19.1-fold higher than that in $\mathrm{FPN1}^{-/+}$and $\mathrm{FPN}^{+/+}$mice, respectively (Fig. 2C). We also found that as FPN1 expression decreased in mouse livers, M2 marker proteins such as TGF- $\beta$ and VEGF were increased significantly, while the expression of $i N O S$, as a M1 marker protein, exhibited the opposite trend (Fig. 2D). The above results indicated that the knockdown of FPN1 in liver cells induced macrophage proliferation and M2 polarization.

FPN1 downregulation in hepatic cells induced hepatic fibrosis. Macrophage polarization is closely related to fibrosis ${ }^{14}$. Immunohistochemical analysis showed that compared to that in the $F P N^{+/+}$group, the expression of Collagen 1, Collagen 4 and matrix metalloproteinase (MMP-9) in $\mathrm{FPN1}^{-{ }^{--}}$and $\mathrm{FPN1}^{-/+}$ mouse livers were enhanced, indicating that the extracellular matrix of liver tissues increased.

Furthermore, additional markers of fibrosis were tested. As expected, the levels of Laminin and $a-S M A$ were increased. Extracellular fibrotic deposition, detected by Sirius red staining and Masson staining, further confirmed that the inhibition of FPN1 impaired hepatic fibrosis in vivo (Fig. 3A-B).

Glutamic-pyruvic transaminase ( $A L T)$ and glutamic oxalacetic transaminase ( $A S T)$ were regards as important index for liver fibrosis or injury. A lots of $A L T$ or $A S T$ are released into the blood and induced increased expression of $A L T$ or $A S T$ in serum when there are hepatocyte necrosis, liver fibrosis or injury. As is shown in Fig. $3 C$ and $D$ further confirm that FPN1 downregulation in hepatic cells induced hepatic fibrosis.

FPN1 regulated macrophage polarization to the M2 phenotype by inducing IL-10 and TGF- $\beta$ expression in vivo. As mentioned above, we found that the downregulation of FPN1 expression in mouse liver cells promoted macrophage $\mathrm{M} 2$ polarization and fibrosis. To investigate which cytokines were involved in this process, Q-PCR and ELISA were used. The Q-PCR results demonstrated that compared to that in $F P N^{+/+}$or $F P N 1^{-/+}$mice, the expression of $I L-1 \beta, I L-4, I L-10, I L-12 \beta, T G F-a, T G F-\beta, H G F, V E G F$, and IFN- $\gamma$ was increased in $F P N 1^{-/-}$hepatocytes (Fig. 4A, B). Though $I L-6, I L-12 a$, TNF- $a$ and CSF-1 showed no significant differences in $F P N 1^{-/-}$and $F P N 1^{-/+}$hepatocytes (Fig. S1-4), the expression of $I L-10$ or TGF- $\beta$ in $\mathrm{FPN1}^{-/-}$hepatocytes showed 5.5-fold or 4.2-fold higher than that in $F P N^{+/+}$hepatocytes. Their ratio was higher than those of IFN- $y$ and $I L-12 \beta$ in $F P N 1^{-/-}$hepatocytes again, which were 3.5-fold and 1.6-fold higher than that in $F P N^{+/+}$hepatocytes, suggesting that FPN1 significantly regulated macrophage polarization to the M2 phenotype, compared with M1 phenotype. Moreover, the ELISA results indicated that the levels of $I L-10, T G F-\beta$, and $I L-4$ were significantly increased in $F P N 1^{-/-}$and $F P N 1^{-/+}$mice (Fig. 4C). These cytokines are strong stimulators of M2 polarization and tissue remodeling and repair ${ }^{15}$. These data suggested that FPN1 induced macrophage polarization and fibrosis through $I L-10$ and TGF- $\beta$.

FPN1 was involved in macrophage polarization in vitro. In order to further confirm the role of FPN1 in macrophage polarization, L02-Scr and L02-Sh cell were constructed by lentivirus vectors with interfere 
FPN1 gene (Fig. 5A-B), this was supported by increase of iron in L02-Sh (Fig. 5C). Interestingly, compared with TPH1 + PMA + L02-SCR group, it is showed for the M2 polarization in TPH1 + PMA + L02-Sh group and TPH1 + PMA + IL-4 + IL-13 group (Fig. 5D), indicated decreased FPN1 promoted liver cancer cell M2 polarization. In addition, the expression of the recognized macrophage marker CD11b and CD68 were analyzed by Western blotting. The number of $C D 68$-positive $\left(C D 68^{+}\right)$cells significantly increased after PMA incubation (Fig. 5E). M0 macrophages were cocultured with L02-SCR and L02-Sh cells. We detected M1 macrophages with an iNOS antibody and M2 macrophages with a CD206 antibody. The in vitro results were consistent with the in vivo results. The expression of CD206 was significantly increased in M0 macrophages cocultured with L02-Sh cells (Fig. 5E), suggesting that macrophages exhibited M2 polarization.

Many reports have indicated that the expression of TNF- $a$ and $I L-6$ are increased in M1 macrophages ${ }^{16}$ and that $I L-10$ and TGF- $\beta$ expression is induced by $M 2$ polarization ${ }^{17,18}$. Thus, we detected the expression levels of these genes in different liver cells and macrophages. The Q-PCR results showed that many cytokines, such as IL-4, IL-10, TGF- $a, T G F-\beta$ and VEGF, were successfully induced in L02-Sh cells compared with L02-SCR cells (Fig. 6A, B; Fig. S5-8). Interestingly, when L02-Sh cells were cocultured with M0 macrophages, the increasing expression of IL-4, IL-10 and TGF- $\beta$ were significantly showed in L02-Sh + M0, compared with L02-SCR + M0, on the contrary, these results were not showed in TNF- $a$ and IFN- $\gamma$, indicated that the high expression of cytokines in the L02-Sh cells promoted M0 to M2 polarization (Fig. 6C), and this was further confirmed by ELISA analysis (Fig. 6D; Fig. S9-11). This indicated decreased FPN1-induced M2 macrophage polarization.

\section{Discussion}

Macrophages are among the most versatile cells in the body ${ }^{19}$. Heterogeneity arises due to macrophage differentiation from monocyte precursors, and the phenotype of macrophages is determined by genetic modification as well as specific tissue-related and immune-related stimuli. Macrophages can be labeled by $C D 68$ or F4/80. M1 macrophages are usually characterized by high expression of CD16/32 and proinflammatory cytokines, including $I L-1 \beta, I L-12$ and TNF-a. M2 macrophages exhibit high expression of CD206 (mannose receptor) and anti-inflammatory cytokines (IL-4, IL-10, TGF- $\beta$, and VEGF). M2 macrophages can secrete profibrotic factors that can promote the activation of myofibroblasts, the synthesis of extracellular matrix, and the development of fibrosis ${ }^{20}$.

The involvement of FPN1 in immune regulation is complex. In recent years, studies have shown that FPN1 is an anti-oncogene in breast cancer and myeloma, but not in the liver 21,22 . Based on our current findings, the downregulation of FPN1 in liver cells promotes M2 macrophage proliferation and polarization in vivo. Moreover, the expression of $\mathrm{M} 2$ markers (CD206, TGF- $\beta$, and VEGF) increased significantly, suggesting that macrophages in the livers of mice had an obvious tendency to polarize towards the M2 phenotype; meanwhile, the expression of CD16/32 and iNOS, as M1 marker proteins, exhibited the opposite trend. Additionally, macrophages were labeled with CD68 and F4/80, and the 
results showed that the number of intrahepatic cells and macrophages increased significantly as FPN1 expression decreased in mouse hepatocytes.

The two main contributors to hepatic fibrosis are excessive fibroblast proliferation and matrix accumulation ${ }^{20}$. The onset of these processes is usually preceded by an acute inflammatory response. Alpha-smooth muscle actin $(a-S M A)$ is a marker of the activation of hepatic stellate cells ${ }^{15}$. Additionally, the activation of hepatic stellate cells can promote transformation into muscle fibrosis cells and ultimately promote the formation of liver fibrosis. TGF- $\beta$ is a known mediator of fibrotic remodeling and matrix accumulation, and the secretion of $T G F-\beta$ by fibroblasts can directly promote collagen synthesis and maturation ${ }^{23}$. This is consistent with the increase in collagen in FPN1 knockout mice. Vascular endothelial growth factor (VEGF) can promote the proliferation of vascular endothelial cells and is also the strongest angiogenic factor. The increased expression of vascular endothelial growth factor in the livers of transgenic mice may be due to hypoxia in local pathological tissues, which stimulates the activation of hepatic stellate cells and then produces a variety of cytokines, resulting in increased expression of vascular endothelial growth factor. Additionally, VEGF can induce endothelial cells to express plasminogen activator and matrix collagenase and then promote peripheral vascular growth ${ }^{15}$. The significant correlation between the levels of VEGF and TGF- $\beta$ in mouse livers is mainly due to the direct activation of vascular endothelial growth factor when the expression of TGF- $\beta$ increases in vivo ${ }^{24}$. Hepatocyte growth factor (HGF) is a multifunctional antifibrotic factor involved in kidney development, acute injury and regeneration ${ }^{25,26}$. Increased expression of HGF and MMP-9 can inhibit ECM production ${ }^{27}$. FPN1 downregulation in hepatic cells increases the expression of Collagen, Laminin, $a-$ $S M A, T G F-\beta$ and $V E G F$, and induces a state of immune tolerance in the livers of mice ${ }^{28}$.

$I L-4, I L-10$ and TGF- $\beta$ can inhibit the expression of TNF- $a, I L-6$ and $I L-12$, leading to the development of a liver microenvironment that favors the $M 2$ polarization of macrophages ${ }^{29,30} . I L-1 \beta$ can attenuate collagen deposition mediated by TGF- $\beta$, which indicates that it has a long-term antifibrotic effect on some tissues ${ }^{31}$. The increased expression of $I L-1 \beta$ may attenuate tissue remodeling mediated by TGF- $\beta$ in the absence of FPN1. Though decreased FPN1 also induce the increase of IFN- $y$ and $I L-12 \beta$, their magnitude of increase was lower than that in $I L-10$ and $T G F-\beta$. This indicated that $M 2$ phenotype macrophages were mainly showed in $F P N 1^{-/-}$mice. In addition, no significant different $T N F-a$ and IFN- $\gamma$ protein expression between L02-Sh and L02-SCR cells is not accordance with significant difference between $F P N 1^{-/-}$mice and $F P N 1^{+/+}$mice, suggested that the protein of TNF- $a$ and IFN- $y$ can easily degraded in vitro. Together, the elevated levels of $I L-4, I L-10$ and $T G F-\beta$ observed in our $F P N 1^{-/-}$animal models may be a response to an increase in M2-mediated remodeling processes that occur in the absence of FPN1.

THP-1 can usually be induced by PMA to obtain macrophages ${ }^{32}$. Furthermore, $I L-4+I L-13$ would transfer M0 to M2 (Fig. S12). Interestingly, Decreased FPN1 not only induced further liver cancer cells M2 polarization, but also increased $C D 206$, indicated $I L-4$ and $I L-13$ cytokines play a key role again. 
Previous studies on FPN1 were mainly related to iron ${ }^{22}$. In this study, we found that FPN1 is also closely related to macrophage proliferation, polarization and fibrosis. Reduced expression of FPN1 in hepatocytes promotes iron accumulation in hepatocytes. This result is not contradiction with previous studies, which have shown that iron-overloaded macrophages exhibit an M1 phenotype ${ }^{33}$, because elevated iron was showed in hepatocytes, or not macrophage in $F P N 1^{-1-}$ mice. Whether it acts macrophage would be further studied. Whether the decrease in FPN1 expression further leads to hepatocellular carcinoma after the formation of the M2 environment in the liver remains to be further studied. It will be very interesting to use conditional knockout mouse models to detect the physiological role of FPN1 in hepatocellular carcinoma in the future.

In conclusion, the downregulation of FPN1 in hepatocytes is beneficial to the proliferation and polarization of macrophages to the M2-like phenotype in the liver, which may lead to fibrosis (Fig. 7). Potential drugs that can upregulate the expression of FPN1 may be new directions in the treatment of hepatic fibrosis.

\section{Materials And Methods}

Cell culture and treatment. THP-1 (Human acute monocytic leukemia cell line) cells and L02 cells were purchased from ATCC. The cells were all cultured in RPMI 1640 containing $10 \%$ heat-inactivated FBS and $1 \%$ penicillin-streptomycin in a $5 \% \mathrm{CO}_{2}$ atmosphere at $37^{\circ} \mathrm{C}$. THP-1 cells were treated with $160 \mathrm{ng} / \mathrm{ml}$ phorbol 12-myristate 13-acetate (PMA) alone and then incubated for $48 \mathrm{~h}$ in RPMI medium to obtain macrophage-like M0 cells. They were then cocultured with L02-SCR or L02-Sh cells in a 6-well Transwell plate.

Animals. FPN $7^{\text {flox/flox }}$ conditional gene knockout mice were constructed by Nancy Andrews of the USA. Two LoxP sites were inserted into exon 6/7 of the SLC4OA1 gene, and the mice were maintained on a 129/SvEvTac background. SLC40A1-LoXPtransgenic mice (129S-Slc40a1tm2Nca/J) were purchased from Jackson Laboratories (USA), backcrossed on a C57BL/ 6 background and bred in-house. When they were bred with liver cell-specific promoter (Alb-Cre) mice, all of the offspring obtained exhibited hepatocyte-specific knockout. Mice with downregulation of the FPN1 gene were used as the model mice for this experiment. Unless indicated, age-matched animals were used at 8-12 weeks of age. Homozygote $\left(F P N 1^{-/-}\right)$, heterozygote $\left(F P N 1^{-/+}\right)$and wild type $\left(F P N 1^{+/+}\right)$mice were obtained by mating, and the genotypes were determined by agarose gel electrophoresis. All animals in this study were raised in SPF-level animal rooms and fed a standard diet. The housing of the animals and the experiment procedures were carried out in accordance with the Guide for the Care and Use of Laboratory Animals (United States National Institutes of Health) and were approved by the Ethical Committee for Care and Use of Laboratory Animals of

Guangzhou Medical University. This study was carried out in compliance with the ARRIVE guidelines 2.0. 
Real-time Quantitative PCR. Total RNA was isolated from THP-1 cells, M0 macrophages, and M0 macrophages cocultured with L02-SCR or L02-Sh using TRIzol according to the manufacturer's instructions. One microgram of RNA was reverse transcribed into cDNA using a First Strand cDNA Synthesis Kit. cDNA was quantified using the Applied Biosystems Step-One Real-Time PCR system with a SYBR Green Real-time PCR Master Mix kit. The following primer sequences that were used are shown in Table 1 (It is showed in supporting information). GAPDH served as the housekeeping gene. 
Table 1

List of the sequence of gene primers.

\begin{tabular}{|c|c|c|}
\hline Gene name & Forward(5'-3') & Reverse(5'-3') \\
\hline IL-1 $\beta$ (mice) & CCAGGATGAGGACATGAGCA & CGGAGCCTGTAGTGCAGTTG \\
\hline IL-4(mice) & CGGCACAGAGCTATTGATGG & TCCGTGGATATGGCTCCTG \\
\hline IL-6(mice) & AGTTGCCTTCTTGGGACTGA & CCTCCGACTTGTGAAGTGGT \\
\hline IL-10(mice) & TAGAGCTGCGGACTGCCTTC & TTCCGATAAGGCTTGGCAAC \\
\hline IL-12a(mice) & CTGGCGTCTACACTGCTGCT & CGTGATTGACACATGCTGGA \\
\hline IL-12ß(mice) & GCTGGTGTCTCCACTCATGG & TCTTCAGGCGTGTCACAGGT \\
\hline TNF-a(mice) & TATGGCTCAGGGTCCAACTC & CCCATTTGAGTCCTTGATGG \\
\hline CSF-1(mice) & CGACTTCCCGTAAAGGCATA & AGCAGAGGGCACTTAAGCAA \\
\hline TGF-a(mice) & CATTGATCTGCCCAGGTCTT & ACTTCTGCCTGGAGCTGTGT \\
\hline TGF- $\beta$ (mice) & TGCCCTCTACAACCAACACA & GTTGGACAACTGCTCCACCT \\
\hline IFN-y(mice) & ССTTTGGACCCTCTGACTTG & AAACAGCCATGAGGAAGAGC \\
\hline HGF(mice) & CTCCCGAGAACTTCAAATGC & GCAGTAGCCAACTCGGATGT \\
\hline VEGF(mice) & СССТTCGTССТСТССТTACС & AAGCCACTCACACACACAGC \\
\hline FPN1 (mice) & GCA GGC TCT GTT CTG GTC CT & GAT GAT TCC GCA GAG GAT GA \\
\hline IL-1 $\beta$ (human) & ACGATGCACCTGTACGATCA & TCTTTCAACACGCAGGACAG \\
\hline IL-4((human) & GCCTTCAGCACATCTTCACACCTC & ATCGCTTCTCTGCACCTGTTCTTG \\
\hline IL-6(human) & GGTGTTGCCTGCTGCCTTCC & GTTCTGAAGAGGTGAGTGGCTGTC \\
\hline IL-10(human) & TGCCTTCAGCAGAGTGAAGA & GTCTTGGTTCTCAGCTTGGG \\
\hline IL-12a(human) & GAGTTCAAGACCAGCCTGACCAAC & АССТССАССТCCGAGTTCAAGC \\
\hline IL-12ß(human) & GAGCAGGCATCAGCACCATCTG & ACACCATCAGCAGCATCACCTTG \\
\hline TNF-a(human) & AGCTGGTGGTGCCATCAGAGG & TGGTAGGAGACGGCGATGCG \\
\hline CSF-1(human) & CAGAAGGAGGACCAGCAAGTGAAG & GCCAGCAAGACCAGGATGACAC \\
\hline TGF-a(human) & GCCTCTGCCGATCTTGAACATCTC & TGCCTACACCTACCTGCTTACCTG \\
\hline TGF- $\beta$ (human) & CACGTGGAGCTGTACCAGAA & GAACCCGTTGATGTCCACTT \\
\hline IFN-y(human) & TGTTACTGCCAGGACCCATA & СTTCCTTGATGGTCTCCACA \\
\hline HGF(human) & AATGGCACGATCTTGGCTCACTG & AGGAGTGGTGGTGGCAGGTG \\
\hline VEGF(human) & TAGCTGCCTGCCTGGTGACTG & CAGAAGGACCACAGGACACAACAC \\
\hline
\end{tabular}




\begin{tabular}{|c|c|c|}
\hline Gene name & Forward(5'-3') & Reverse(5'-3') \\
\hline FPN1(human) & TGA ATG CCA CAA TAC GAA GG & CCA AGT TCC ATC CCG AAA TA \\
\hline$\beta$-Actin & GCC ACT GCC GCA TCC TCT TC & AGC CTC AGG GCA TCG GAA CC \\
\hline 14146 & GGC ATT CCC AAC ACT TTA GC & \\
\hline 14147 & CCC ATA GGT TAA ACT GCT TCA A & \\
\hline 20239 & TGCAAACATCACATGCACAC & \\
\hline 20240 & TTGGCCCCTTACCATAACTG & \\
\hline olMR5374 & GAAGCAGAAGCTTAGGAAGATGG & \\
\hline
\end{tabular}

ELISA. The levels of IL-4, IL-10, TGF- $\beta$, TNF- $a$, and IFN- $\gamma$ (Cell signaling Company, USA) in the serum and cell culture supernatant were measured by ELISA following the manufacturer's instructions. All ELISA reagent kits were purchased from ExCellBio. All samples were measured in triplicate. The concentration of certain cytokines in the serum and cell culture was quantified by standard curve. The fold change in the expression of cytokines was calculated compared to the control group.

Agarose Gel Electrophoresis. Agarose Gel Electrophoresis was performed as described previously ${ }^{34}$. DNA was extracted using a GeneJET Genomic DNA Purification kit (Thermo, USA). Extracted DNA samples were amplified by PCR of the FPN1 gene by restriction fragment length polymorphism-PCR. The PCR mixture contained $12 \mu \mathrm{L}$ of OneTaq Quick-Load 2X Master Mix with standard buffer, $4 \mu \mathrm{L}$ of each primer $(40 \mu \mathrm{M})$, and $8 \mu \mathrm{L}$ of DNA sample $(<10 \mathrm{ng})$, resulting in a total volume of $28 \mu \mathrm{L}$. The reaction was carried out under the following conditions: initial denaturation at $95^{\circ} \mathrm{C}$ for $15 \mathrm{~min}$ followed by 34 cycles of denaturation at $95{ }^{\circ} \mathrm{C}$ for $1 \mathrm{~min}$, annealing at $56{ }^{\circ} \mathrm{C}$ for $1 \mathrm{~min}$ and elongation at $72{ }^{\circ} \mathrm{C}$ for $1 \mathrm{~min}$ with a final extension at $72{ }^{\circ} \mathrm{C}$ for $10 \mathrm{~min}$. Gel electrophoresis was performed on $1.5 \sim 2 \%$ agarose gels supplemented with $11 \mathrm{mM} \mathrm{MgCl}_{2}$ and SYBER ${ }^{\text {TM }}$ safe DNA gel stain in TAE buffer $(0.5 \times)$ at pH 8 . The samples of interest were mixed with $20 \%$ loading buffer $(6 x)$ and then loaded into agarose gel wells. The gel was run at $70 \mathrm{~V}$ for $2.5-3 \mathrm{~h}$ and visualized using a UVP scanner.

Western Blot. The cells were washed with ice-cold PBS and then lysed in ice-cold RIPA lysis buffer containing $1 \mathrm{mmol} / \mathrm{L}$ PMSF. The protein concentrations were calculated using BCA assay kits. A total of $40 \mu \mathrm{g}$ of total cellular protein was subjected to $8 \%$ SDS-PAGE and transferred to a PVDF membrane. The membrane was blocked with $5 \%$ defatted milk powder at room temperature for $1.5 \mathrm{~h}$, incubated with primary antibodies (FPN1, CD11b, CD206, iNOS, and GAPDH were come from Cell Signaling Company, USA) at $4^{\circ} \mathrm{C}$ overnight and then incubated with HRP-conjugated secondary antibodies at room temperature for $1.5 \mathrm{~h}$. Following each step, the membranes were washed five times with TBS-T for 5 min. Finally, the blots were developed using the enhanced chemiluminescence system.

Immunohistochemical analysis. Immunohistochemical analysis was played according to the procedure reported by Hau-Wen $\mathrm{Li}^{35}$. Deparaffinization of the tissue sections was executed using a graded ethanol 
series, and then $0.3 \%$ hydrogen peroxide was used to block endogenous peroxidase activity. Antigen retrieval was achieved by placing the sections in $10 \mathrm{mmol} / \mathrm{L}$ citrate buffer $(\mathrm{pH} 6.0)$ and heating in an autoclave for $5 \mathrm{~min}$. The sections were then rinsed with phosphate-buffered saline (PBS, pH 7.2) and incubated with $10 \%$ nonimmunized goat serum for $30 \mathrm{~min}$ at room temperature to inhibit nonspecific binding. The tissue sections were then incubated at $4^{\circ} \mathrm{C}$ overnight with antibodies. After $24 \mathrm{~h}$, the slides were incubated with horseradish peroxidase (HRP)-conjugated anti-mouse/rabbit secondary antibodies for $30 \mathrm{~min}$ at $25^{\circ} \mathrm{C}$. Then, staining with 3,3-diaminobenzidine (DAB) was carried out for 90 seconds at room temperature; hematoxylin staining was performed as a control.

Histology. Tissue from mice was fixed with $4 \%$ paraformaldehyde, embedded in paraffin, and sectioned (thickness $=5 \mu \mathrm{m}$ ). Masson staining was conducted by a Masson Stain Kit (Solarbio, Beijing, China). Sirius red staining was performed to visualize collagen fibers using a Sirius Red Stain Kit (Solarbio, Beijing, China). Each section was assessed under light microscopic fields.

Flow cytometry. $\mathrm{F} 4 / 80^{+}, C D 16 / 32^{+}$, and $C D 206^{+}$cells were quantified by antibody labeling and flow cytometry. Briefly, cells were dispersed with $0.25 \%$ EDTA-trypsin and washed with PBS. For each group, $10^{6}$ cells were resuspended in $500 \mu \mathrm{lPBS}$ and labeled with $5 \mu \mathrm{l}$ of antibody. The cells were incubated at room temperature for $30 \mathrm{~min}$ and resuspended 2-3 times during incubation. The data were acquired on an LSRII and analyzed by FlowJo v8.8.6.

Antibodies and reagents. The antibody to FPN1 (\#L3266) was obtained from Santa Cruz Biotechnology (USA). The antibody to GAPDH (\#5174) was obtained from cell signaling Company (USA). The antibody to Collagen-1 (\#ab34710), Collagen-4 (\#ab6586), Laminin (\#ab11575), MMP9 (\#ab38898), a-SMA (\#ab5694), VEGF(\#ab46154), TGF-B(\#ab92486), INOS(\#ab15323) were obtained from abcam (USA). The antibody to F4/80 (\#123107) and CD206 (\#141706) were obtained from Biolegend. The antibody to F4/80(\#12-4801-82), CD68(\#11-0689-42) and CD16/32 (\#4303632)were obtained from Invitrogen. Sirus Red Staining Kit (\#G1471) was obtained from Solarbio (China) and Masson Staining Kit (\#D026-1-3) was obtained from NanJing JianCheng (China)

Iron assay. The relative iron concentration in cell lysates was assessed using an Iron Assay Kit (\#ab83366, Abcam) according to the manufacturer's instructions.

Statistical analysis. Statistical analysis was performed using GraphPad Prism software version 6.0. All data are expressed as the mean \pm standard deviation (SD). Comparisons between two groups were performed using Student's $t$ test, and three or more groups were evaluated for significance using one-way ANOVA combined with Bonferroni's post hoc test. A $P$ value $<0.05$ or 0.001 considered statistically significant.

\section{Declarations}

\section{Data Availability}


The datasets used in the current study are available from the corresponding authors on reasonable request.

\section{Ethical Approval}

The housing of the animals and the experiment procedures were carried out in accordance with the Guide for the Care and Use of Laboratory Animals (United States National Institutes of Health) and were approved by the Ethical Committee for Care and Use of Laboratory Animals of Guangzhou Medical University. This article does not contain any studies with human participants performed by any of the authors.

\section{Nonstandard abbreviations}

$I L-1 \beta$, interleukin $1 \beta ; / L-4$, interleukin 4; $I L-6$, interleukin 6; $I L-10$, interleukin $10 ; / L-12 a$, interleukin $12 a ; / L-$ $12 \beta$, interleukin-12 $\beta$; TNF- $a$, tumor necrosis factor- $\alpha$; $C S F-1$, macrophage colony-stimulating factor; TGF-a, transforming growth factor- $\alpha$; $T G F-\beta$, transforming growth factor- $\beta$; IFN- $\gamma$, interferon $\gamma ; H G F$, hepatocyte growth factor; $V E G F$, vascular endothelial growth factor; $F P N 1$, ferroportin.

\section{Author contributions}

He Wang and Yi Zhou designed the experiments. He Wang and Chengyuan Cai wrote the manuscript; Qing Gao, Chengyuan Cai, and Danning Zeng performed the experiments; Bohang Zeng and Lei Ma analyzed data; He Wang supervised the project. All authors reviewed the manuscript.

\section{Acknowledgements}

This study was supported by 2018 High-level university academic backbone training program in Guangzhou Medical University(B185004199), 2018 Guangdong Key discipline construction project of pharmacy (Q185031010), 2019 Undergraduate laboratory open project(C195015003), 2018 Construction of scientific research teaching and academic improvement project in Guangzhou Medical University (B185004025), and 2017 University innovation and strengthening program (Q17024031).

\section{Competing interests}

The authors declare no conflicts of interest.

\section{References}

1. Rockey, D. C., Bell, P. D. \& Hill, J. A. Fibrosis - A Common Pathway to Organ Injury and Failure. New Engl J Med. 372, 1138-1149 https://doi.org/10.1056/NEJMra1300575 (2015).

2. Donovan, A. et al. The iron exporter ferroportin/SIc40a1 is essential for iron homeostasis \%JCell Metabolism.1 (2005). 
3. Deicher, R. \& Horl, W. H. New insights into the regulation of iron homeostasis. Eur J Clin Invest. 36, 301-309 https://doi.org/10.1111/j.1365-2362.2006.01633.x (2006).

4. Pietrangelo, A. The ferroportin disease.

5. Pietrangelo, A. Ferroportin disease: pathogenesis, diagnosis and treatment. Haematologica. 102, 1972-1984 https://doi.org/10.3324/haematol.2017.170720 (2017).

6. Pietrangelo, A. et al. Hereditary hemochromatosis in adults without pathogenic mutations in the hemochromatosis gene.

7. Gautier, E. L. \& Yvan-Charvet, L. Understanding macrophage diversity at the ontogenic and transcriptomic levels.

8. Daemen, S. \& Schilling, J. D.The Interplay Between Tissue Niche and Macrophage Cellular Metabolism in Obesity.

9. Baker, A. D. et al.Targeted PPAR\{gamma\} deficiency in alveolar macrophages disrupts surfactant catabolism.

10. Krenkel, O. \& Tacke, F. Liver macrophages in tissue homeostasis and disease.

11. Wynn, T. A. \& Vannella, K. M. Macrophages in Tissue Repair, Regeneration, and Fibrosis.

12. Mantovani, A., Sica, A., Fau - Locati, M. \& Locati, M.Macrophage polarization comes of age.

13. Sabelli, M. et al. Human macrophage ferroportin biology and the basis for the ferroportin disease.

14. Tang, P. M., Nikolic-Paterson, D. J. \& Lan, H. Y. Macrophages:versatile players in renal inflammation and fibrosis.

15. Vannella, K. M. \& Wynn, T. A.Mechanisms of Organ Injury and Repair by Macrophages.

16. Kroner, A. et al. TNF and increased intracellular iron alter macrophage polarization to a detrimental M1 phenotype in the injured spinal cord.

17. Toossi, Z. et al. Enhanced production of TGF-beta by blood monocytes from patients with active tuberculosis and presence of TGF-beta in tuberculous granulomatous lung lesions.

18. Fulton, S. A., Toossi, C. J. F. \& Boom, W. H. Z. T., Toossi Zt Fau - Boom, W. H. \& Regulation of interleukin-12 by interleukin-10, transforming growth factor-beta, tumor necrosis factor-alpha, and interferon-gamma in human monocytes infected with Mycobacterium tuberculosis H37Ra.

19. Gordon, S. \& Taylor, P. R. Monocyte and macrophage heterogeneity.

20. Ramachandran, P. \& Iredale, J. P. Macrophages:central regulators of hepatic fibrogenesis and fibrosis resolution.

21. Gu, Z. et al. Decreased ferroportin promotes myeloma cell growth and osteoclast differentiation.

22. Pinnix, Z. K. et al.Ferroportin and iron regulation in breast cancer progression and prognosis.

23. Vashaghian, M. et al. Gentle cyclic straining of human fibroblasts on electrospun scaffolds enhances their regenerative potential.

24. Yan, Z. et al. CD147 promotes liver fibrosis progression via VEGF-A/VEGFR2 signalling-mediated cross-talk between hepatocytes and sinusoidal endothelial cells. 
25. Ejaz, A., Epperly, M. W., Hou, W., Greenberger, J. S. \& Rubin, J. A. O. Adipose-Derived Stem Cell Therapy Ameliorates lonizing Irradiation Fibrosis via Hepatocyte Growth Factor-Mediated Transforming Growth Factor- $\beta$ Downregulation and Recruitment of Bone Marrow Cells.

26. Borovikova, A. A. et al. Adipose-Derived Tissue in the Treatment of Dermal Fibrosis: Antifibrotic Effects of Adipose-Derived Stem Cells.

27. Li, G. et al. Macrophage-secreted TSLP and MMP9 promote bleomycin-induced pulmonary fibrosis.

28. Williams, M. J., Clouston, A. D. \& Forbes, S. J. Links between hepatic fibrosis, ductular reaction, and progenitor cell expansion.

29. Huang, Y. et al. IL-16 regulates macrophage polarization as a target gene of mir-145-3p.

30. Huang, Z. et al. IL-37 Expression is Upregulated in Patients with Tuberculosis and Induces Macrophages Towards an M2-like Phenotype.

31. Siwik, D. A., DI Fau, C., Colucci, W. S. \& Colucci, W. S. - \& Interleukin-1 beta and tumor necrosis factoralpha decrease collagen synthesis and increase matrix metalloproteinase activity in cardiac fibroblasts in vitro.

32. Nold, M. F. et al.IL-37 is a fundamental inhibitor of innate immunity.

33. Zhou, Y. A. O. et al. Iron overloaded polarizes macrophage to proinflammation phenotype through ROS/acetyl-p53 pathway.

34. Xing, Y. et al. Raman observation of a molecular signaling pathway of apoptotic cells induced by photothermal therapy. Chemical science. 10, 10900-10910 https://doi.org/10.1039/c9sc04389f (2019).

35. Li, H. W. et al. The Characteristics and Expression Profile of Transferrin in the Accessory Nidamental Gland of the Bigfin Reef Squid during Bacteria Transmission. Scientific reports. 9, 20163 https://doi.org/10.1038/s41598-019-56584-8 (2019).

\section{Figures}




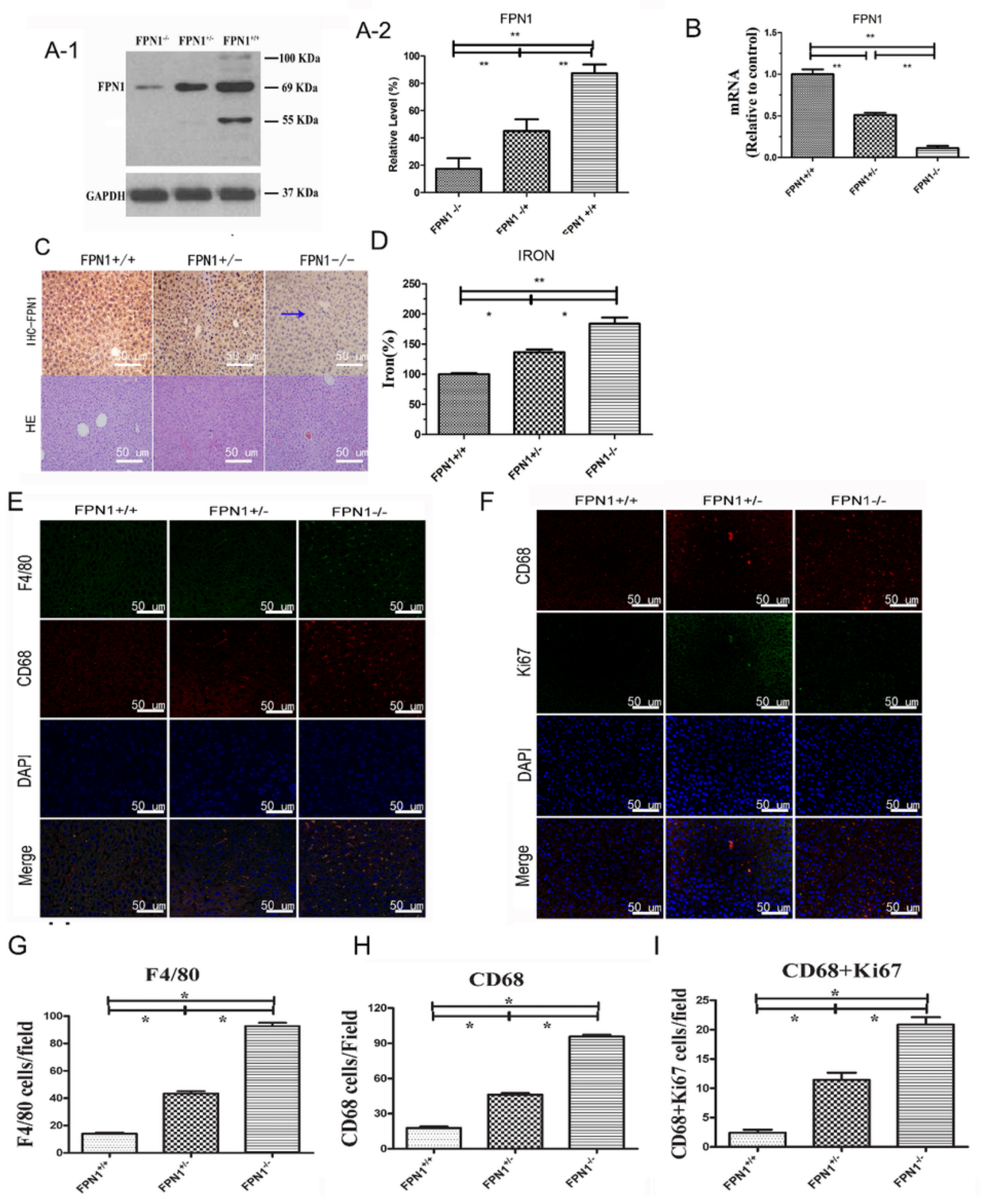

\section{Figure 1}

Downregulation of FPN1 in hepatic cells induced macrophage proliferation in vivo. Western blot (A), QPCR (B), HE staining and immunohistochemical (C) analysis of the expression of FPN1 in hepatocytes of transgenic mice. (D) The iron content in mouse livers (FPN1+/+, FPN1-/+, and FPN1-/-). (E) F4/80 (green) and CD68 (red) staining of liver sections. Total nuclei were costained with DAPI (blue). (F) Ki67 (green) and F4/80 (red) staining of liver sections. Total nuclei were costained with DAPI (blue) $(\times 400)$. 
Quantification analysis of F4/80-positive (G), and CD68-positive (H) nuclei and F4/80+Ki67-positive (I). All results are from three independent experiments. ${ }^{*} \mathrm{P}<0.05,{ }^{*} \mathrm{P}<0.001$.
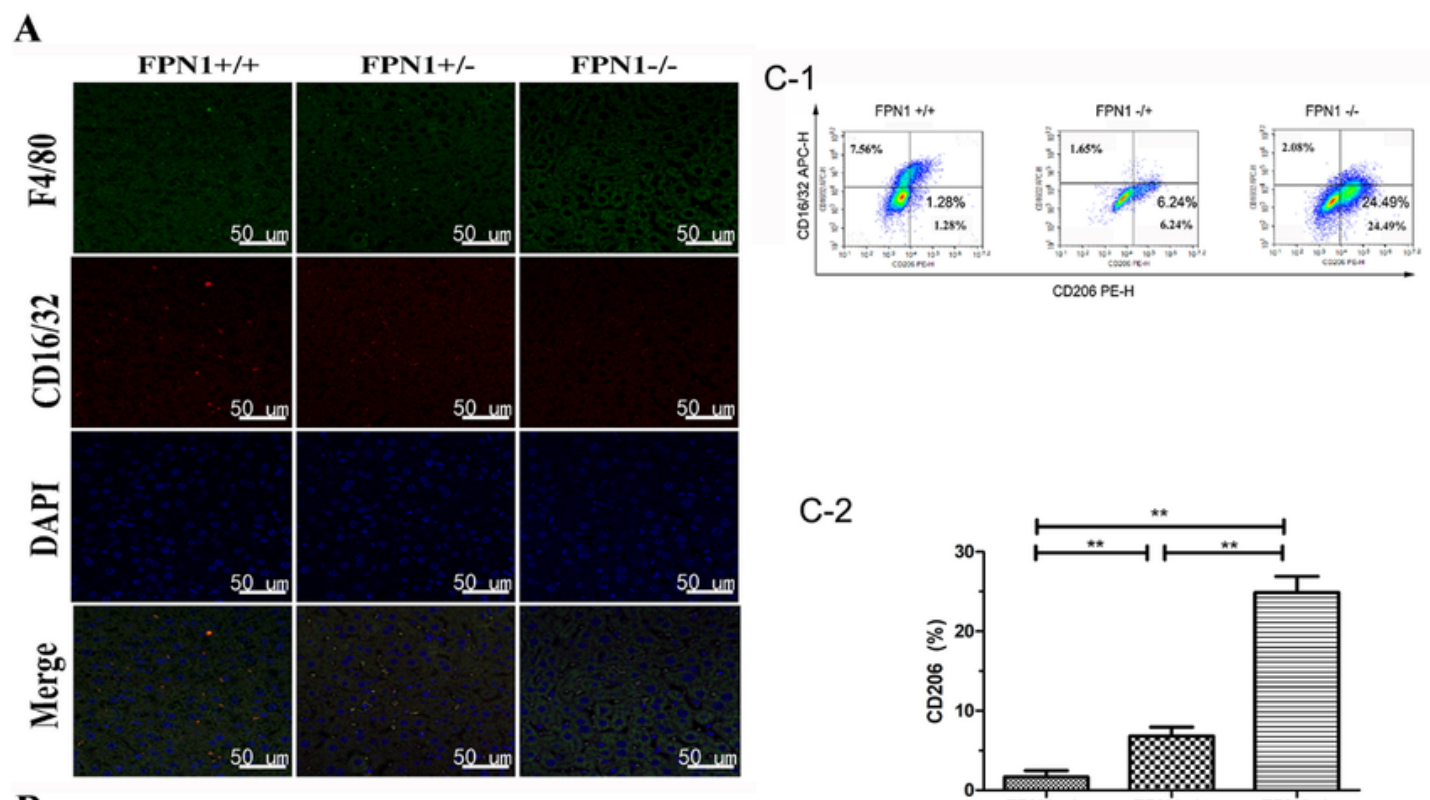

B

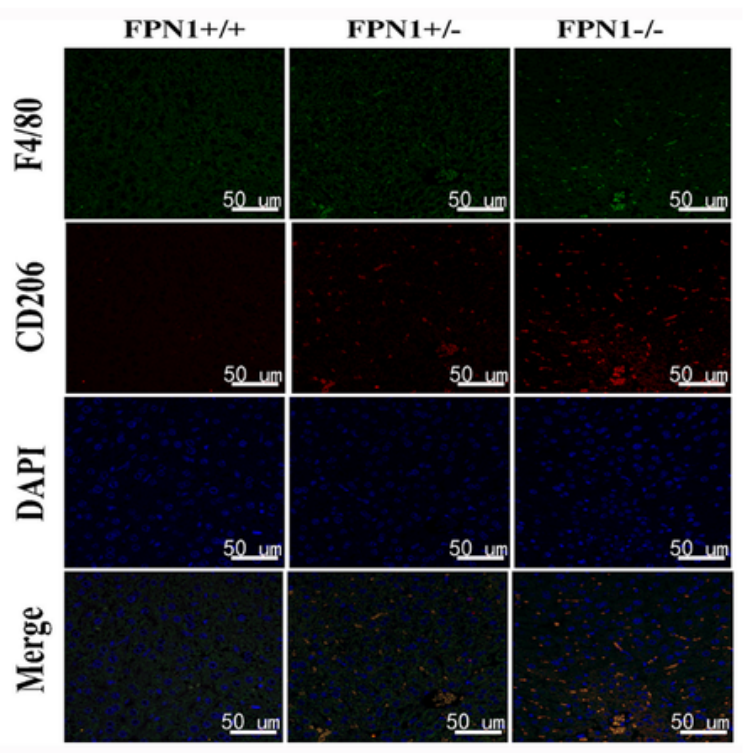

C-2

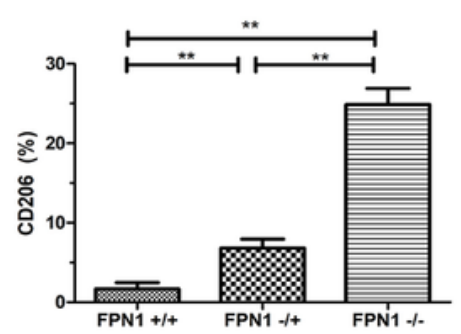

D-2
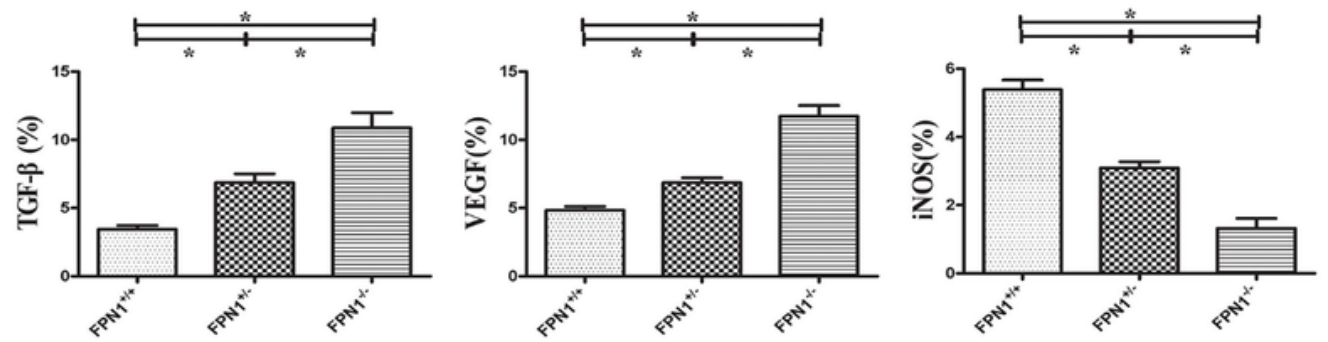

Figure 2

Downregulation of FPN1 in liver cells promoted M2 macrophage polarization in vivo. Liver sections were co-stained with F4/80 (A) and CD206 or CD16/32 (B). M1 macrophages were F4/80high and CD16/32high, and M2 macrophages were F4/80high and CD206high. (C) FCM analysis of the expression 
of specific biomarkers of macrophages in mouse livers. (1) Image of FCM; (2) Apoptosis level of FCM. (D) $\mathrm{IHC}$ analysis of the expression of M1 and M2 marker proteins in mouse livers. (1) Image of IHC; (2) TGF- $\beta$, VEGF and iNOS level of IHC. All results are from three independent experiments. ${ }^{*} P<0.05,{ }^{* * P}<0.001$.

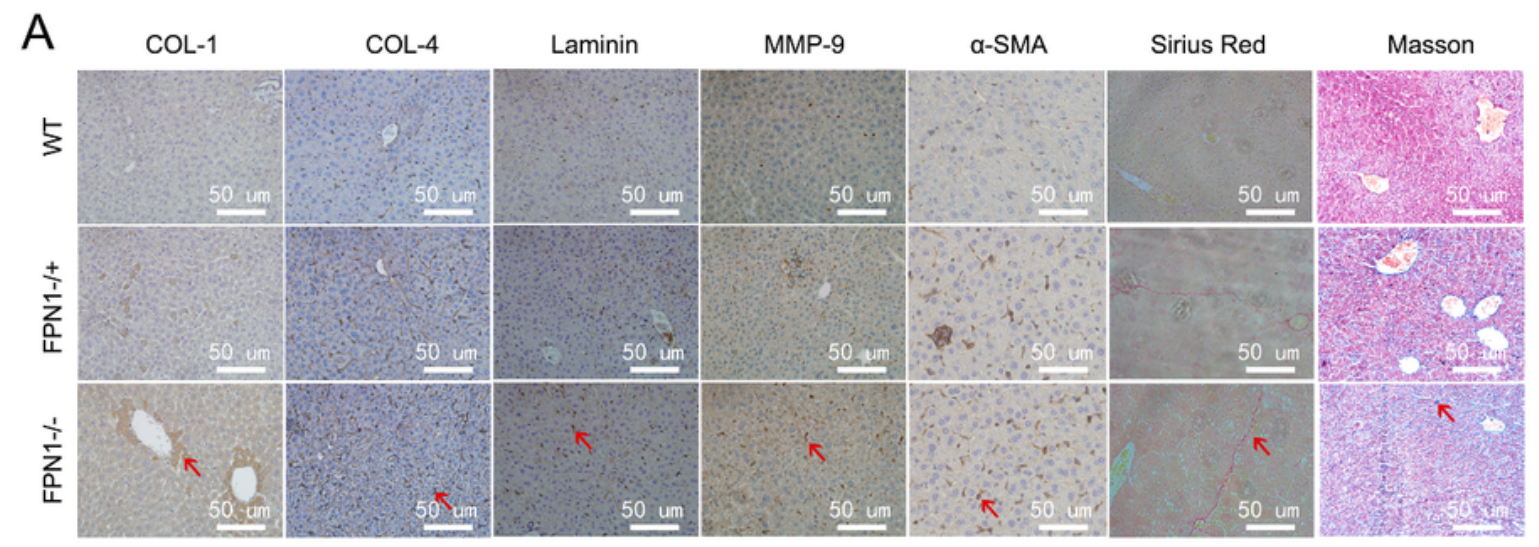

B
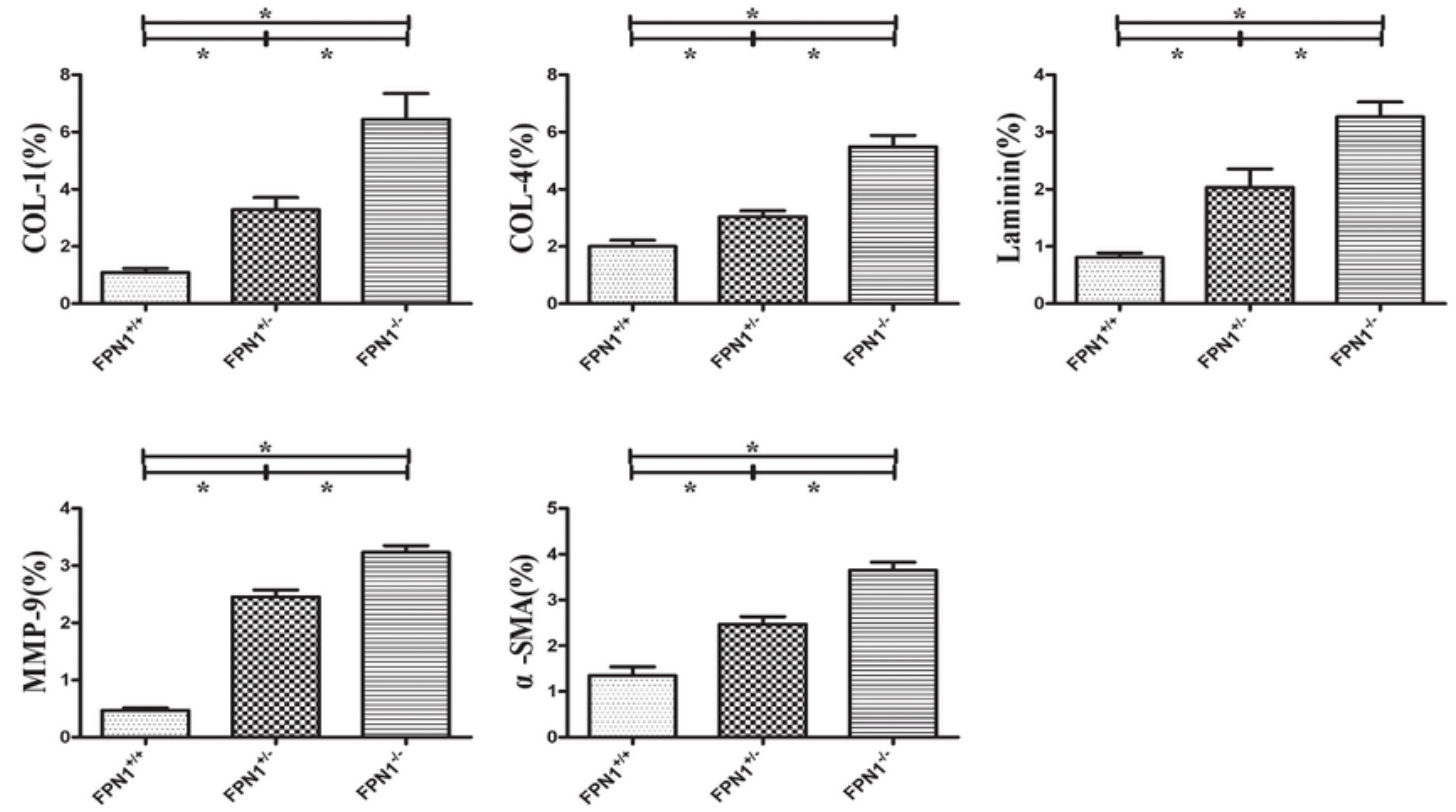

C
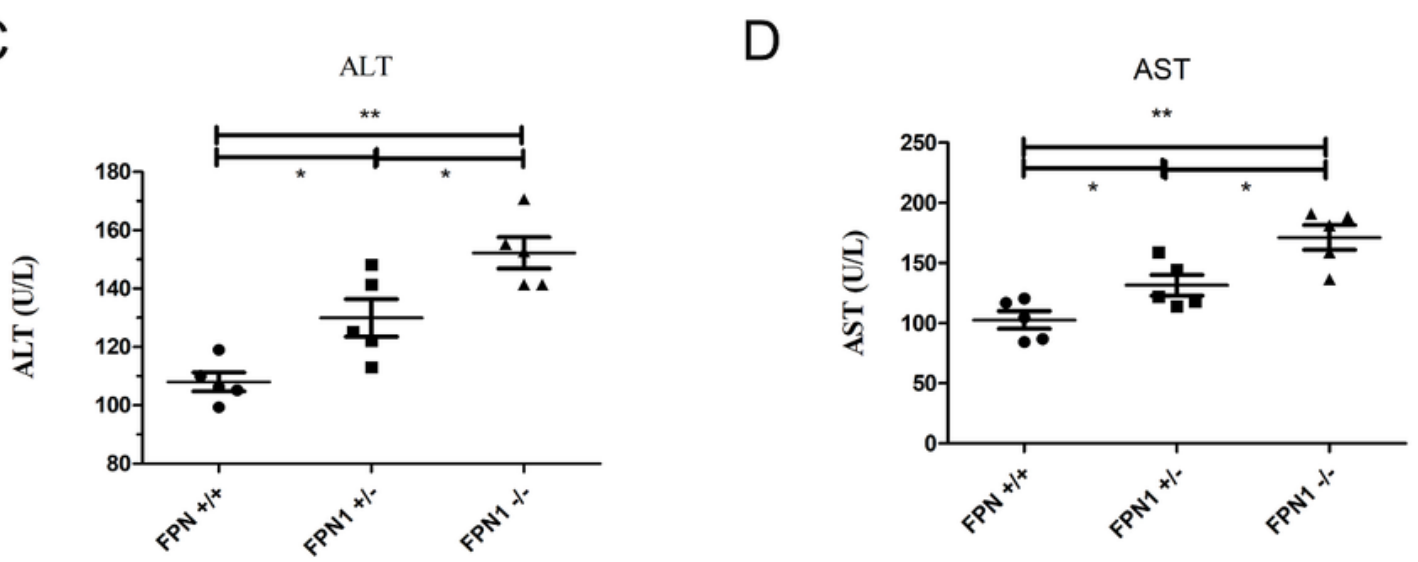

Figure 3

Downregulation of FPN1 in hepatic cells induced liver fibrosis. (A) Liver sections were incubated with antibodies against COL-1, COL-4, Laminin, a-SMA, and MMP-9 and stained with Sirius red or Masson ( $\times$ 
400). (B) Error bars correspond to the mean \pm standard deviation of IHC. ALT (C) and AST (D) content in mouse serum (FPN1+/+, FPN1-/+, FPN1-/-). All results are from three independent experiments. ${ }^{*} \mathrm{P}<0.05$, $\star * \mathrm{P}<0.001$.

A-1

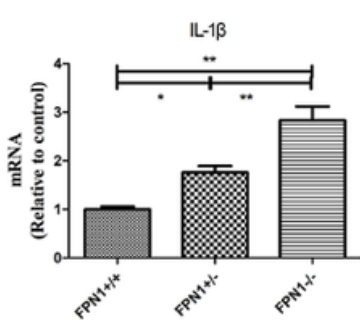

A-3

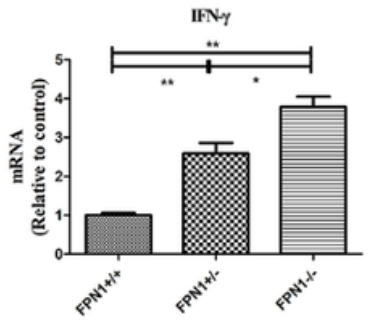

B-2

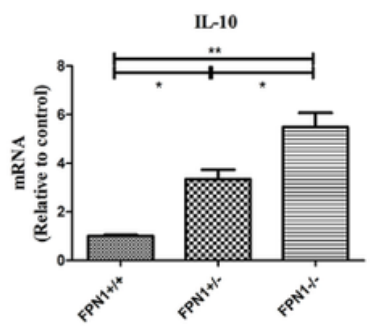

A-2

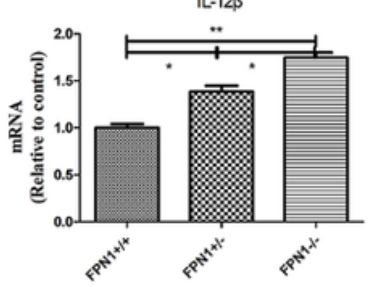

B-1

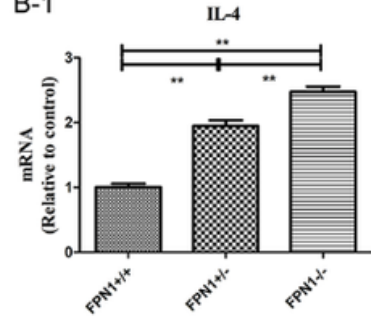

B-3

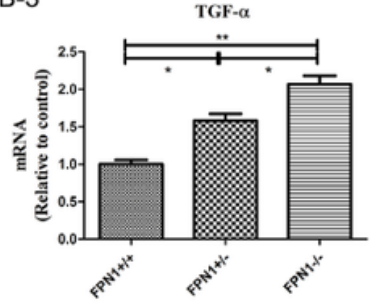

B-4

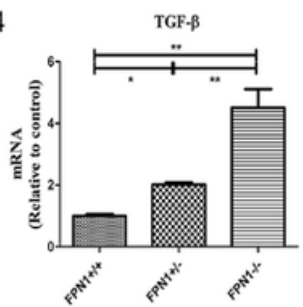

C-1

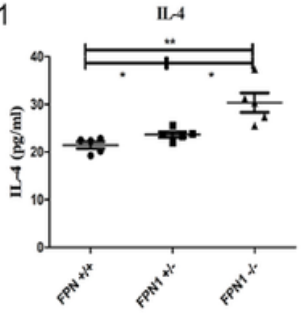

C-4

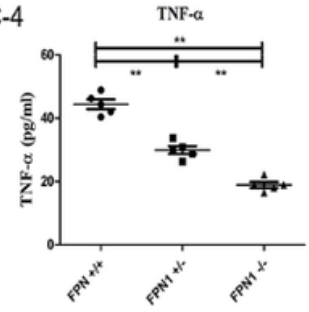

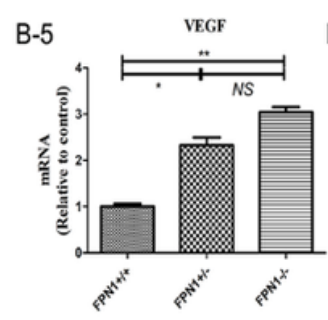

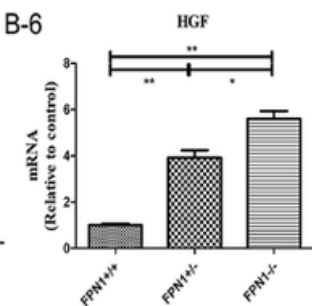

C-2

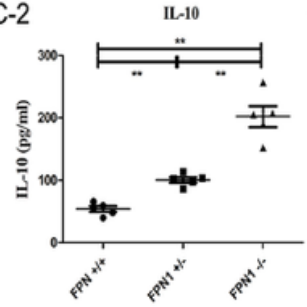

C-3

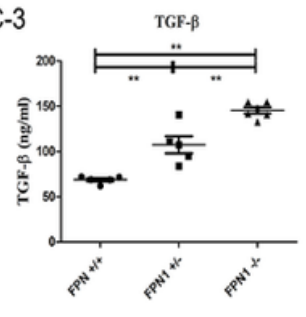

C-5

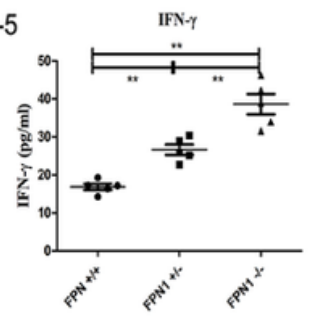

Figure 4

FPN1 downregulation in hepatic cells increased the expression of specific biomarkers of M2 macrophages in vivo. (A, B) Q-PCR analysis of the expression of specific biomarkers in mouse livers. (C) ELISA analysis of the expression of specific biomarkers in mouse serum. All results are from three independent experiments. ${ }^{*} \mathrm{P}<0.05,{ }^{\star} * \mathrm{P}<0.001$. 
A-1

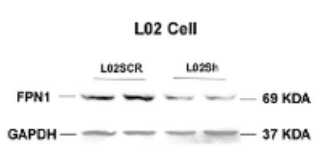

B

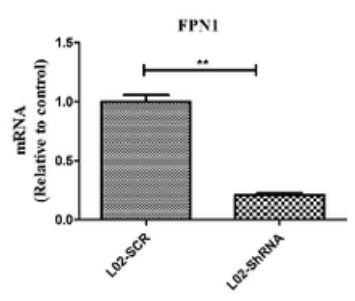

D

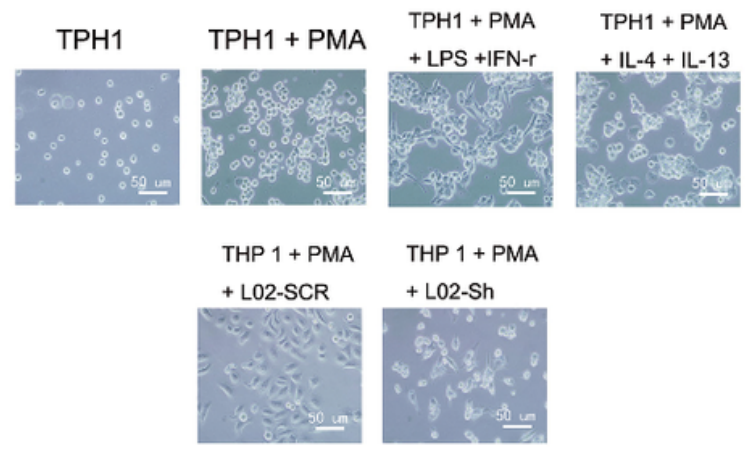

A-2 FPN1
$\mathrm{E}-1$

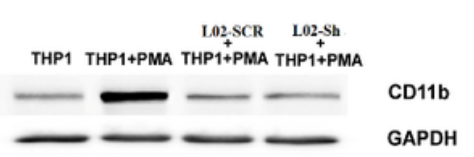

C
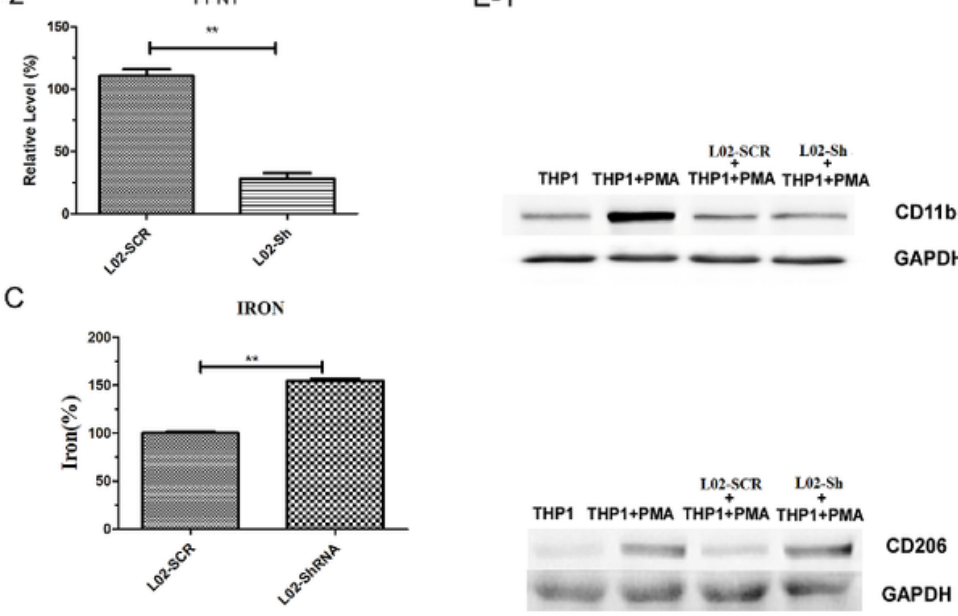

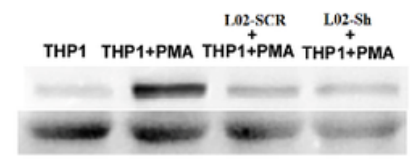

E-2
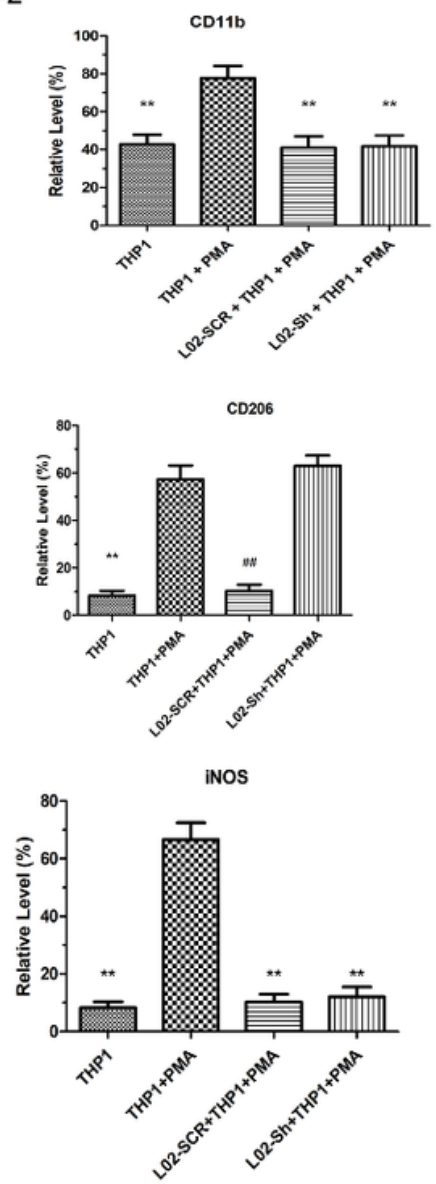

\section{Figure 5}

Downregulation of FPN1 in L02 cells promoted M2 macrophage polarization in vitro. Western blot (A) and Q-PCR (B) analysis of the expression of FPN1 in L02-SCR and L02-Sh cells. (C) Iron content in L02-SCR and L02-Sh cells. (D) THP-1 cells plated in culture plates were incubated with $160 \mathrm{nM}$ PMA in RPMI 1640 medium containing $5 \%$ FBS for $48 \mathrm{~h}$. For M1 macrophage induction, $10 \mathrm{pg} / \mathrm{ml} \mathrm{LPS}$ and $20 \mathrm{ng} / \mathrm{ml} \mathrm{IFN- \gamma}$ were added to the medium without PMA. For M2 macrophage induction, $20 \mathrm{ng} / \mathrm{ml} \mathrm{IL-4}$ and $20 \mathrm{ng} / \mathrm{ml} \mathrm{IL-}$ 13 were added to the medium. (E) Western blot analysis of CD68, CD11b, CD206 and iNOS expression in macrophages. (1) Image of western blot; (2) Grey level of western blot. All results are from three independent experiments. ${ }^{*} \mathrm{P}<0.001$. ${ }^{*} \mathrm{P}<0.001$, vs THP1 + PMA. \#\# P<0.001, vs L02-Sh + THP1 + PMA 

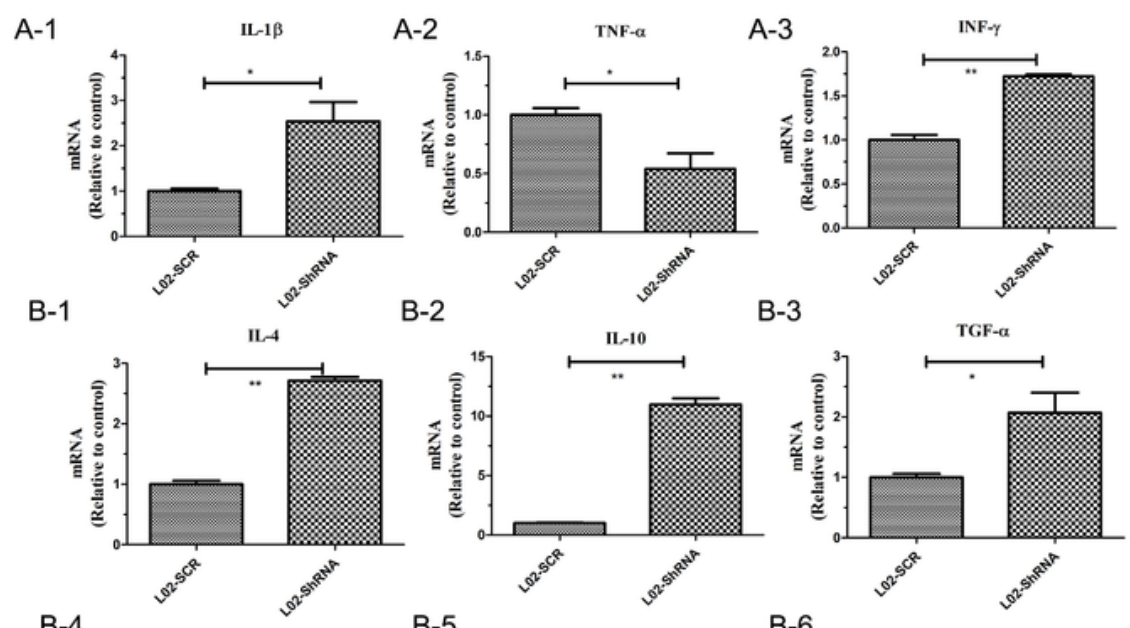

B-2

B-3
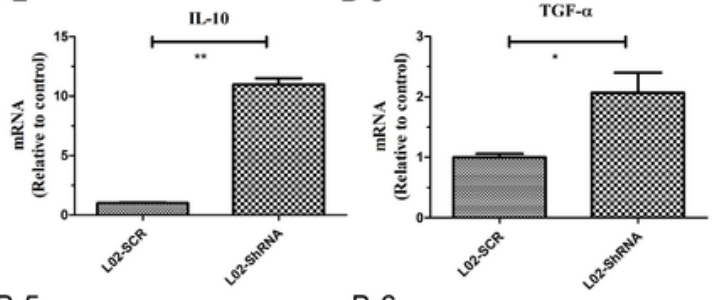

B-4

B-5

B-6
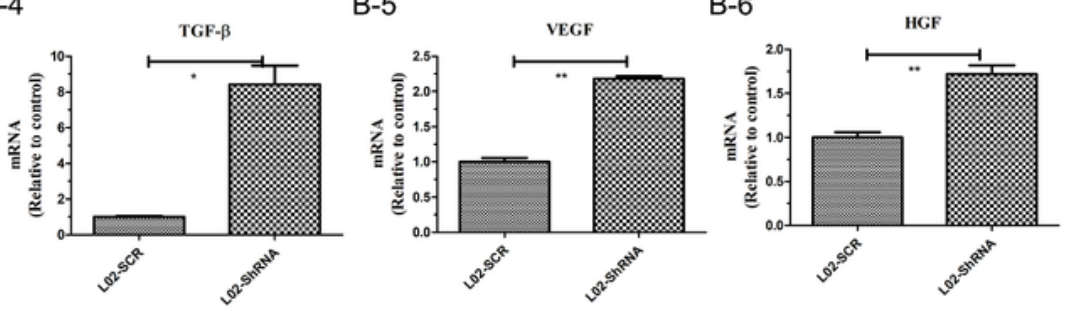

C-1
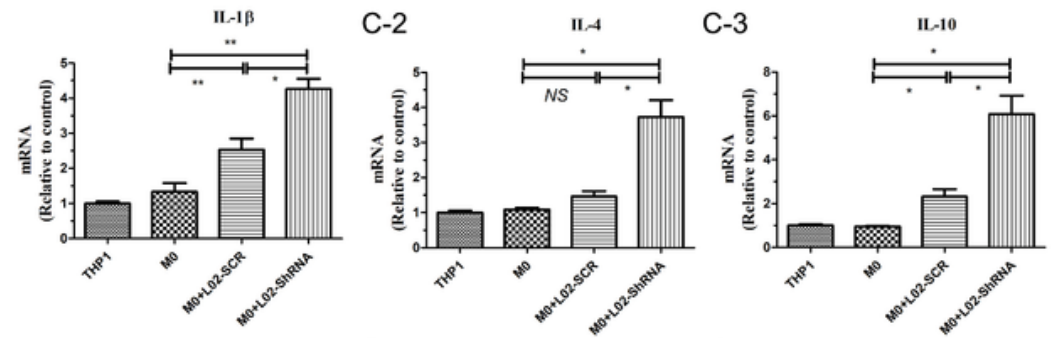

C-4

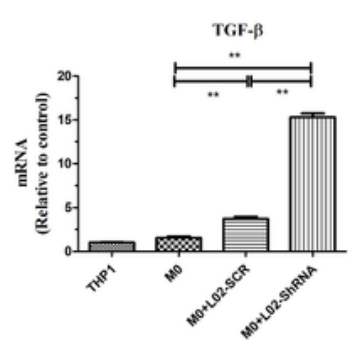

C-5

C-6

D-1
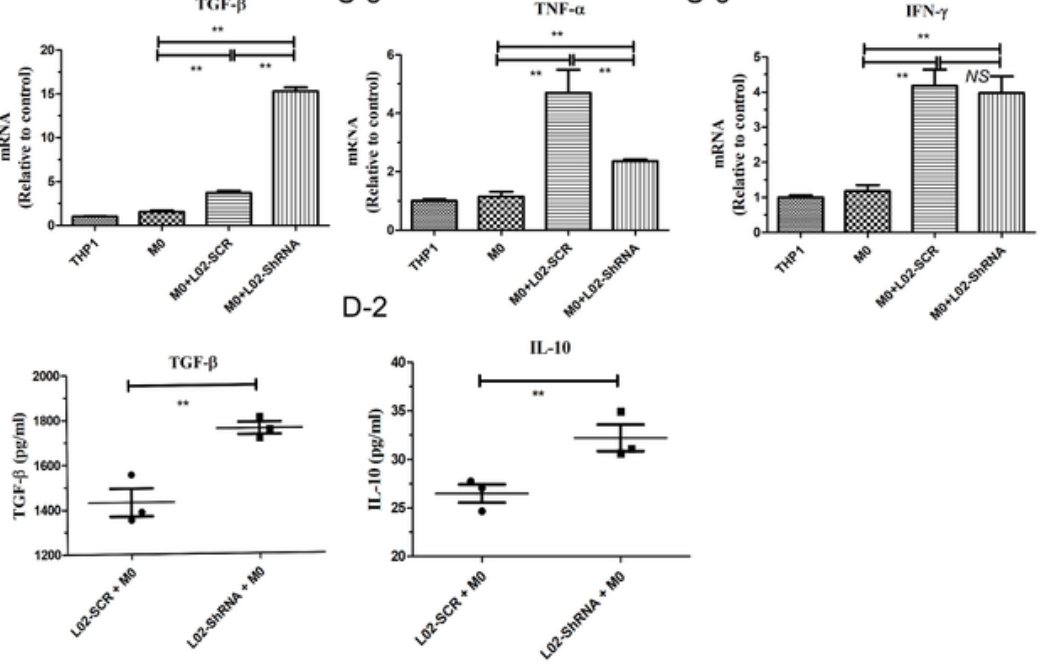

\section{Figure 6}

Silencing FPN1 increased the expression of specific biomarkers of $M 2$ macrophages in vitro. $(A, B$ and $C)$ Q-PCR analysis of the expression of specific biomarkers in L02-Sh cells, L02-SCR cells+M0 macrophages, and L02-Sh cells +M0 and macrophages. (D) ELISA analysis of the expression of specific biomarkers in the culture medium. All results are from three independent experiments. ${ }^{*} P<0.05,{ }^{\star \star} P<0.001$. 


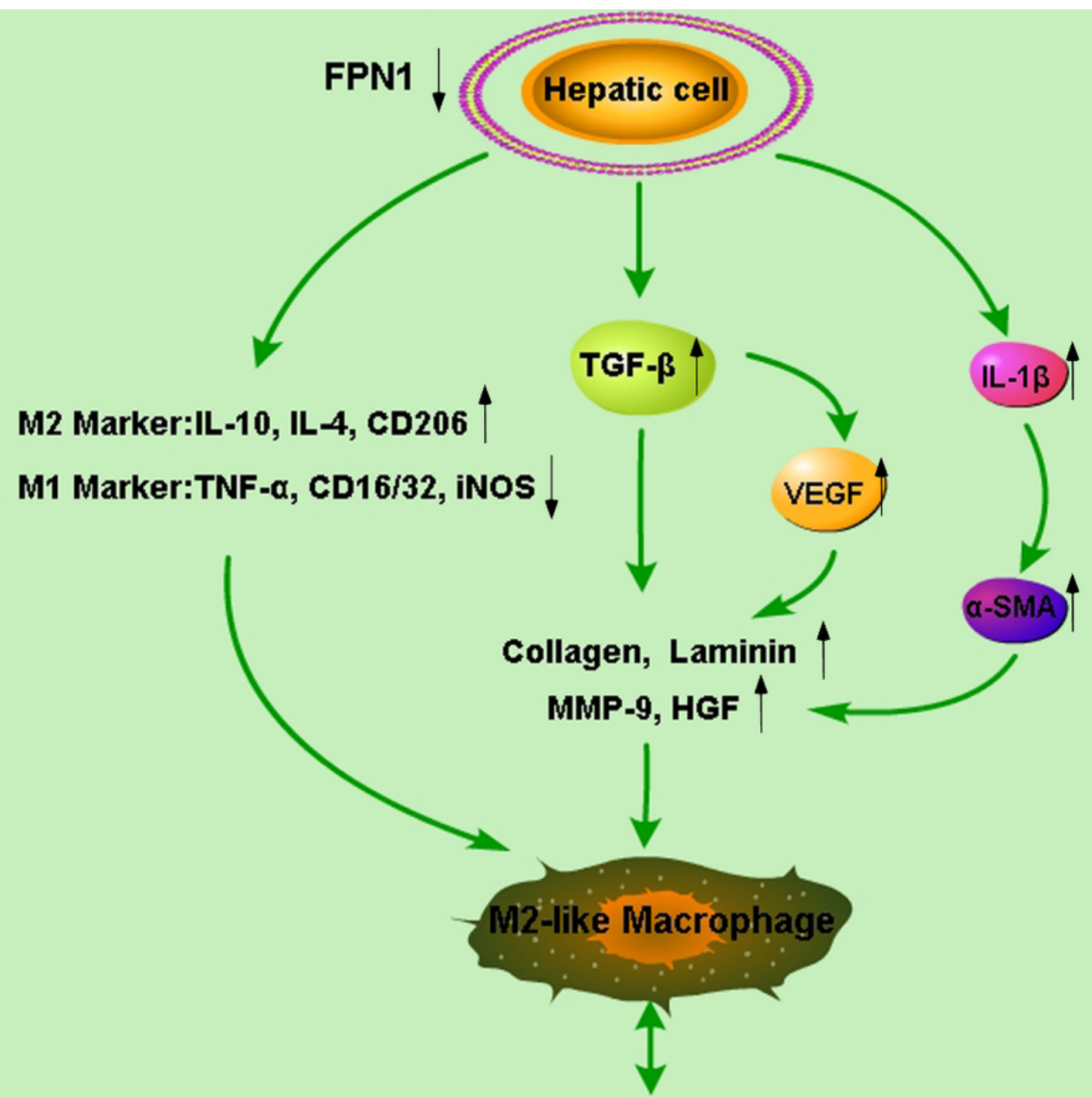

Fibrosis

Figure 7

Molecular mechanism of FPN1 involvement in macrophage polarization and hepatic fibrosis. Downregulation of FPN1 in hepatic cells increases the levels of IL-10 and TGF- $\beta$, thus inducing macrophage polarization to the M2 phenotype while promoting hepatic fibrosis.

\section{Supplementary Files}

This is a list of supplementary files associated with this preprint. Click to download.

- SupportingInformation.docx 